\title{
Global extraction of the jet transport coefficient in cold nuclear matter
}

\author{
Peng Ru๑, ${ }^{1,2}$ Zhong-Bo Kang, ${ }^{3,4}$ Enke Wang, ${ }^{1,2, *}$ Hongxi Xing $\odot,{ }^{1,2, \dagger}$ and Ben-Wei Zhang ${ }^{5}$ \\ ${ }^{1}$ Guangdong Provincial Key Laboratory of Nuclear Science, Institute of Quantum Matter, \\ South China Normal University, Guangzhou 510006, China \\ ${ }^{2}$ Guangdong-Hong Kong Joint Laboratory of Quantum Matter, \\ Southern Nuclear Science Computing Center, South China Normal University, Guangzhou 510006, China \\ ${ }^{3}$ Department of Physics and Astronomy, University of California, Los Angeles, California 90095, USA \\ ${ }^{4}$ Mani L. Bhaumik Institute for Theoretical Physics, University of California, \\ Los Angeles, California 90095, USA \\ ${ }^{5}$ Key Laboratory of Quark \& Lepton Physics (MOE) and Institute of Particle Physics, \\ Central China Normal University, Wuhan 430079, China
}

(Received 3 August 2019; revised 30 May 2020; accepted 8 February 2021; published 26 February 2021)

\begin{abstract}
Within the framework of the generalized QCD factorization formalism, a set of nuclear-dependent observables all arise from the quark-gluon and gluon-gluon correlation functions, which are closely connected to the well-known jet transport coefficient $(\hat{q})$ for the nucleus. In this paper, we perform the first global analysis of $\hat{q}$ for cold nuclear matter with a kinematics dependent parametrization. The analysis takes into account the world data on transverse momentum broadening in semi-inclusive electron-nucleus deep inelastic scattering, Drell-Yan dilepton and heavy quarkonium production in proton-nucleus collisions, as well as the nuclear modification of the structure functions in deep inelastic scattering, comprising a total of 215 data points from 8 datasets. Within our scheme, we clarify quantitatively the universality and kinematics dependence of the nuclear medium property as encoded in $\hat{q}$. We expect that the determined parametrization of $\hat{q}$ in cold nuclear matter will have significant impact on precise identification of the transport property of hot dense medium created in heavy ion collisions.
\end{abstract}

DOI: 10.1103/PhysRevD.103.L031901

\section{INTRODUCTION}

The exploration of the inner structure and properties of nuclear medium is of fundamental importance in nuclear science. In relativistic heavy ion collisions, of particular interest is the jet transport coefficient $\hat{q}$. Physically, $\hat{q}$ represents the transverse momentum broadening per unit length of an incoming jet encountered in a hot and dense medium, and describes the interaction strength between the hard probe and nuclear medium. In recent years, the jet transport coefficient $\hat{q}$ has become a standard quantity in searching for and to characterize the properties of the quark-gluon plasma [1]. $\hat{q}$ is the key quantity in the study of jet quenching phenomena and parton energy loss, as it arises in all the theoretical descriptions of energetic probes in heavy ion collisions [2-4], which is remarkable.

\footnotetext{
wwangek@scnu.edu.cn

†hxing@m.scnu.edu.cn
}

Published by the American Physical Society under the terms of the Creative Commons Attribution 4.0 International license. Further distribution of this work must maintain attribution to the author(s) and the published article's title, journal citation, and DOI. Funded by SCOAP ${ }^{3}$.
So far there have been significant efforts and a lot of progress in extracting $\hat{q}$ for hot dense medium through measurements of jet quenching at Relativistic Heavy Ion Collider (RHIC) and the Large Hadron Collider (LHC) [1,5-11]. On the other hand, a comprehensive study of the transport property of cold nuclear matter is still lacking, which we set out to address in this paper. Significant theoretical progress also makes now an opportune time to perform a detailed study with the theoretical inputs. For example, for a long time, $\hat{q}$ has been assumed to be a constant in most studies [12-14]. However, this is inconsistent with recent theoretical developments, where renormalization group equations for $\hat{q}$ have been derived through explicit calculations of radiative corrections [15-20]. In particular, complete next-to-leading order (NLO) calculations of transverse momentum broadening for real scattering processes have shown the universality and scale dependence of the twist-4 quark-gluon correlation functions for the nucleus [15-17], which in turn implies the universality and scale-dependence of the corresponding $\hat{q}$ in the nuclear medium. However, the obtained evolution equation therein is not closed, thus requires computation at even higher orders, which is unfortunately formidable in the current quantitative study of scale dependence of the medium property. 
In this paper, we aim at a data driven understanding of the medium property of cold nuclear matter based on well defined physical observables and rigorous QCD factorization formalism, and thus to address how/whether the experimental data provide useful information on the $\hat{q}$. It has long been recognized that the phenomenon of transverse momentum broadening, defined as the difference of averaged transverse momentum square between nuclear and hadronic collisions, can be treated as an excellent observable to probe the nuclear medium property and the QCD multiple scattering dynamics [21,22]. Experimentally, significant transverse momentum broadening effects have been observed in various experiments involving different identified final state particles at different collision energies and collision systems [23-31]. Theoretically, this observable has been systematically calculated within the rigorous theoretical framework of the generalized factorization formalism [12,32-35], or the high-twist expansion approach, in which one can attribute the transverse momentum broadening to the next-to-leading power correction in the momentum transfer, and the size is determined by the twist -4 partonparton correlation functions in the nucleus.

It has been shown that $\hat{q}$ in semi-inclusive electronnucleus deep inelastic scattering (SIDIS) and Drell-Yan (DY) have the same functional form and satisfy the same QCD evolution equation. The experimental check of the universality of nuclear medium property would provide a critical test of the generalized factorization formalism. In this letter, we will carry out, for the first time, a combined fit of world data on transverse momentum broadening in SIDIS [23], DY dilepton and heavy quarkonium production in proton-nucleus (pA) collisions [24-30]. Notice that the dynamical shadowing effect as observed in electronnucleus (eA) deep inelastic scattering (DIS) is also sensitive to the value of $\hat{q}$ [13], therefore we include Fermilab E665 data [36,37] into our analysis as well. From our global analysis presented below, we provide a quantitative evidence of the universality and kinematic dependence of the nuclear medium property within our scheme.

\section{TRANSVERSE MOMENTUM BROADENING AND $\hat{q}$}

The nonperturbative but universal $\hat{q}$ can be accessed in measurements of transverse momentum broadening. Taking SIDIS as an example, the transverse momentum broadening is defined as $\Delta\left\langle p_{T}^{2}\right\rangle=\left\langle p_{T}^{2}\right\rangle_{\mathrm{eA}}-\left\langle p_{T}^{2}\right\rangle_{\mathrm{ep}}$, with $p_{T}$ the transverse momentum of final state hadron and $\left\langle p_{T}^{2}\right\rangle_{\mathrm{eA} / \mathrm{ep}}$ the average transverse momentum in eA (or ep) collisions. The averaged transverse momentum square is an inclusive observable and perturbatively calculable as the transverse momentum is integrated over. The first nontrivial leading contribution comes from final state double scattering manifested as twist- 4 power corrections to the cross section [38]
$\Delta\left\langle p_{T}^{2}\right\rangle=\frac{4 \pi^{2} \alpha_{s} z_{h}^{2}}{N_{c}} \frac{\sum_{q} e_{q}^{2} T_{q g}\left(x_{B}, 0,0, \mu^{2}\right) D_{h / q}\left(z_{h}, \mu^{2}\right)}{\sum_{q} e_{q}^{2} f_{q / A}\left(x_{B}, \mu^{2}\right) D_{h / q}\left(z_{h}, \mu^{2}\right)}$,

where $f_{q / A}\left(x_{B}, \mu^{2}\right)$ is the parton distribution function with $x_{B}$ the Bjorken- $x$, and $D_{h / q}\left(z_{h}, \mu^{2}\right)$ is the hadron fragmentation function with $z_{h}$ the momentum fraction. The twist-4 quark-gluon correlation function is defined as

$$
\begin{aligned}
T_{q g}(x, 0,0)= & \int \frac{d y^{-}}{2 \pi} e^{i x p^{+} y^{-}} \int \frac{d y_{1}^{-} d y_{2}^{-}}{4 \pi} \theta\left(y_{2}^{-}\right) \theta\left(y_{1}^{-}-y^{-}\right) \\
& \times\left\langle A\left|\bar{\Psi}_{q}(0) y^{+} F_{\alpha}^{+}\left(y_{2}^{-}\right) F^{\alpha+}\left(y_{1}^{-}\right) \Psi_{q}\left(y^{-}\right)\right| A\right\rangle,
\end{aligned}
$$

which contains the fundamental properties of the nuclear medium as probed by a propagating quark. Under the approximation of a large and loosely bound nucleus, one can neglect the momentum and spatial correlations of two nucleons. Thus $T_{q g}\left(x, 0,0, \mu^{2}\right)$ can be effectively factorized as [39]

$$
T_{q g}\left(x, 0,0, \mu^{2}\right) \approx \frac{9 R_{A}}{8 \pi^{2} \alpha_{s}} f_{q / A}\left(x, \mu^{2}\right) \hat{q}(x, \mu),
$$

where $R_{A}$ is the nuclear radius, $\hat{q}(x, \mu)$ is the nuclear geometry averaged quark jet transport coefficient and is assumed to be independent of $A$.

In the Drell-Yan process, the transverse momentum broadening of final state dileptons is caused purely by initial state multiple scattering. The final expression is analogous to Eq. (1), except that one replaces the fragmentation functions with parton distribution functions for the beam proton $[38,40]$. In heavy quarkonium production in pA collisions, the transverse momentum broadening receives contributions not only from the initial state multiple scattering analogy to that in Drell-Yan process, but also the final state double scattering between the heavy quark pair and nuclear medium. Details of the calculation and final expressions can be found in $[41,42]$ for both the color evaporation model and nonrelativistic QCD effective theory.

Last but not least, resummed power corrections to the DIS nuclear structure functions $F_{2}\left(x_{B}, Q^{2}\right)$ have been calculated systematically in the framework of the pQCD factorization approach with resummed higher twist contributions [13]. It is found that the shadowing effect as observed in experiment is also sensitive to the value of $\hat{q}$, therefore provide us with another type of good observable to probe the nuclear medium property. All these calculations are performed within the same collinear factorization framework, i.e., high-twist expansion.

\section{GLOBAL ANALYSIS AND RESULTS}

The idea of the global analysis is to extract the nonperturbative functions entering the factorized cross sections. This technique has been extensively used to explore the 
nucleon 1D and 3D structures, in which parametrized forms of the nonperturbative functions are assumed for the global analysis. Similarly, we adopt the following flexible functional form to parametrize $\hat{q},{ }^{1}$

$$
\hat{q}\left(x, \mu^{2}\right)=\hat{q}_{0} \alpha_{s}\left(\mu^{2}\right) x^{\alpha}(1-x)^{\beta} \ln ^{\gamma}\left(\mu^{2} / \mu_{0}^{2}\right),
$$

where $\alpha_{s}\left(\mu^{2}\right)$ is introduced to offset the strong coupling constant $\alpha_{s}$ in the denominator of Eq. (3), and $\mu_{0}=1 \mathrm{GeV}$ is introduced to make the argument in the logarithm dimensionless. The $\ln \left(\mu^{2} / \mu_{0}^{2}\right)$-dependence is motivated by the radiative corrections of $\hat{q}[18,19,44]$. Thus, we have 4 free parameters $\hat{q}_{0}, \alpha, \beta$, and $\gamma$ to be fitted to experimental data. The term $\ln ^{\gamma}\left(\mu^{2} / \mu_{0}^{2}\right)$ represents any deviation in the QCD evolution of $T_{q g}\left(x, 0,0, \mu^{2}\right)$ from that of $f_{q / A}\left(x, \mu^{2}\right)$, see Eq. (3), and thus mimics the scale-dependence of $\hat{q}$ to be determined from the experimental data. In the small- $x$ region, we expect $\hat{q}$ to be proportional to the gluon saturation scale $Q_{s}^{2} \propto x^{-1 / 3}$ [45] and thus the factor $x^{\alpha}$ in $\hat{q}$. Finally in the large- $x$ region, power corrections could also be different $[46,47]$ and thus we have the factor $(1-x)^{\beta}$. We use the MINUIT package [48] to perform a global fit of the $\hat{q}$ from world data. To be consistent with the region of applicability of collinear factorization formalism, we include only the data points with $Q^{2}>1 \mathrm{GeV}^{2}$. Notice that the nuclear dynamic shadowing datasets from CERN-NA37 have been excluded due to the disagreement with those from FNAL-E665 datasets, and we choose FNAL-E665 datasets as they provide better fit of $\hat{q}$.

In this analysis, due to the lack of complete NLO calculations of transverse momentum broadening in eA and $\mathrm{pA}$ collisions, we stick to $\mathrm{LO}$ of $\mathrm{pQCD}$ results, where only diagonal twist- 4 matrix elements are involved. We leave those involving off-diagonal twist- 4 matrix element, such as energy loss calculations in eA $[49,50]$ and pA collisions [51], for future works when more data is available for reasonable constraints. As for the proton PDFs, we use CT14 at LO with $n_{f}=3$ active quark flavors [52]. For pion PDFs, we take the parametrization form as in Ref. [53]. As for fragmentation functions, we use the DSS parametrization [54]. For heavy quarkonium, we set heavy quark mass $m_{c}=1.5 \mathrm{GeV}, m_{b}=4.5 \mathrm{GeV}$. We set the renormalization and factorization scale the same $\mu_{r}^{2}=\mu_{f}^{2}=Q^{2}$, with $Q$ the invariant mass of the virtual photon or heavy quarkonium mass. The uncertainties due to initial state leading-twist nonperturbative functions and final state heavy quarkonium production mechanism are largely canceled as the transverse momentum broadening is a ratio of the transverse momentum weighted and the total cross sections.

We now present results for our global analysis of SIDIS, DY, heavy quarkonium in $\mathrm{pA}$ and structure functions in eA.

\footnotetext{
${ }^{1}$ An unbiased determination of $\hat{q}$ could be achieved via neutral network approach [43].
}

TABLE I. Data used in our analysis, the individual and total $\chi^{2}$ values of the fit. We employ cuts of $Q>1 \mathrm{GeV}$ for the DIS structure function for reliable pQCD calculation.

\begin{tabular}{lrcc}
\hline \hline Experiment & $\begin{array}{c}\text { Data } \\
\text { points }\end{array}$ & $\begin{array}{c}\chi^{2} \\
\text { (const. } \hat{q})\end{array}$ & $\begin{array}{c}\chi^{2} \\
{\left[\hat{q}\left(x, Q^{2}\right)\right]}\end{array}$ \\
\hline HERMES [23] & 156 & 218.5 & 189.7 \\
FNAL-E772 [27] & 4 & 2.69 & 1.65 \\
SPS-NA10 [31] & 5 & 6.86 & 6.47 \\
FNAL-E772 [24,28] & 4 & 2.33 & 2.67 \\
FNAL-E866 [25,27] & 4 & 2.03 & 2.45 \\
RHIC [29] & 10 & 44.4 & 31.0 \\
LHC [30] & 12 & 87.3 & 4.8 \\
FNAL-E665 [36,37] & 20 & 23.7 & 21.46 \\
TOTAL: & 215 & 387.9 & 260.2 \\
\hline \hline
\end{tabular}

In Table I, we list all the datasets that are included in our analysis as well as their respective $\chi^{2}$ values with 4 free parameters in the fit. In total we have fitted 215 data points from 8 datasets. Shown in Fig. 1 is the kinematic reach in existing data. The capability to probe $\hat{q}$ is mainly located in the intermediate $x_{B}$ and $Q^{2}$ region. Future measurements with wider kinematic coverage (e.g., at a future Electron Ion Collider) is indispensable for a complete understanding of medium property.

In order to clarify the $x$ and scale $\mu^{2}$ dependence of $\hat{q}\left(x, \mu^{2}\right)$, we follow the usual way to treat $\hat{q}$ as a constant in the fit, i.e., setting $\alpha=\beta=\gamma=0$ and eliminating $\alpha_{s}$ dependence in Eq. (4). As expected and advocated above, with just one free parameter $\hat{q}_{0}$, the MINUIT fit fails to converge particularly in small- $x$ region to reach a minimized $\chi^{2}$. To elaborate further, we perform the fit for individual datasets, and present the fitted $\hat{q}$ in each individual process in Fig. 2. One can immediately conclude that the fitted $\hat{q}$ for different processes (or same process but in different kinematic regions) are not a single constant, and can even differ by a factor of two or more. Apparently this commonly used fit of constant $\hat{q}$ contradicts with the statement that $\hat{q}$ is universal as stated in Ref. [15].

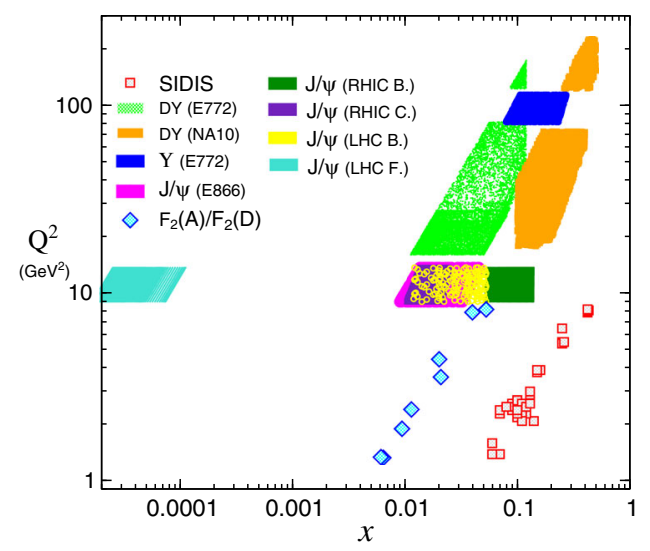

FIG. 1. The range in Bjorken- $x$ and $Q^{2}$ accessible in existing data. 


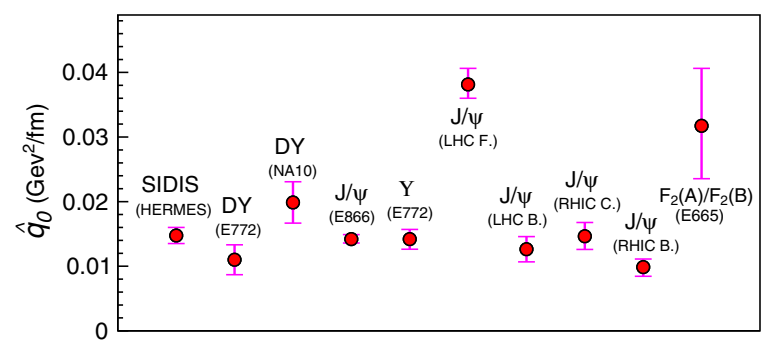

FIG. 2. The fitted $\hat{q}$ from each individual process with parameters $\alpha=\beta=\gamma=0$ in Eq. (4). "F.", "B." and "C." represent for "forward," "backward," and "central" rapidity, respectively. Uncertainties from the fits are indicated by the vertical bars.

This exercise thus hints that there would be nontrivial $x$ and $\mu^{2}$ dependence of $\hat{q}$.

In this paper, we aim to overcome this unsatisfactory situation jeopardizing the reliability of generalized factorization formalism. We perform a global fit to all relevant high-quality data from eA and $\mathrm{pA}$ collisions with 4 free parameters. Specifically, the $x_{B}, \nu, Q^{2}$ dependence in
SIDIS, DY, and heavy quarkonium would provide good constraints on the parametrization form of $\hat{q}$, and the atomic number $A$ distribution or number of binary collisions $N_{\text {coll }}$ dependence will serve as a strong check of higher twist expansion approach as it predicts that multiple scattering leads to linear medium size dependence of transverse momentum broadening. In Fig. 3, we compare our theoretical results with some representative datasets, including transverse momentum broadening in SIDIS, transverse momentum broadening for DY lepton pair and heavy quarkonium in $\mathrm{pA}$ collisions, and nuclear modification ratio $F_{2}(A) / F_{2}(D)$ in the DIS structure function $F_{2}$. The solid curve corresponds to the best fit with $\hat{q}_{0}=0.0195_{-0.0065}^{+0.0085} \mathrm{GeV}^{2} / \mathrm{fm}, \quad \alpha=-0.174_{-0.069}^{+0.077}, \quad \beta=$ $-2.79_{-2.04}^{+1.99}$, and $\gamma=0.254_{-0.166}^{+0.174}$, while the shaded area corresponds to the uncertainty in the global fit with 90\% confidence level. Standard Lagrange multiplier technique $[55,56]$ is employed in order to assess the uncertainty of the $\hat{q}$ determined in the fit. The uncertainties of the theoretical predictions shown in Fig. 3 vary with the
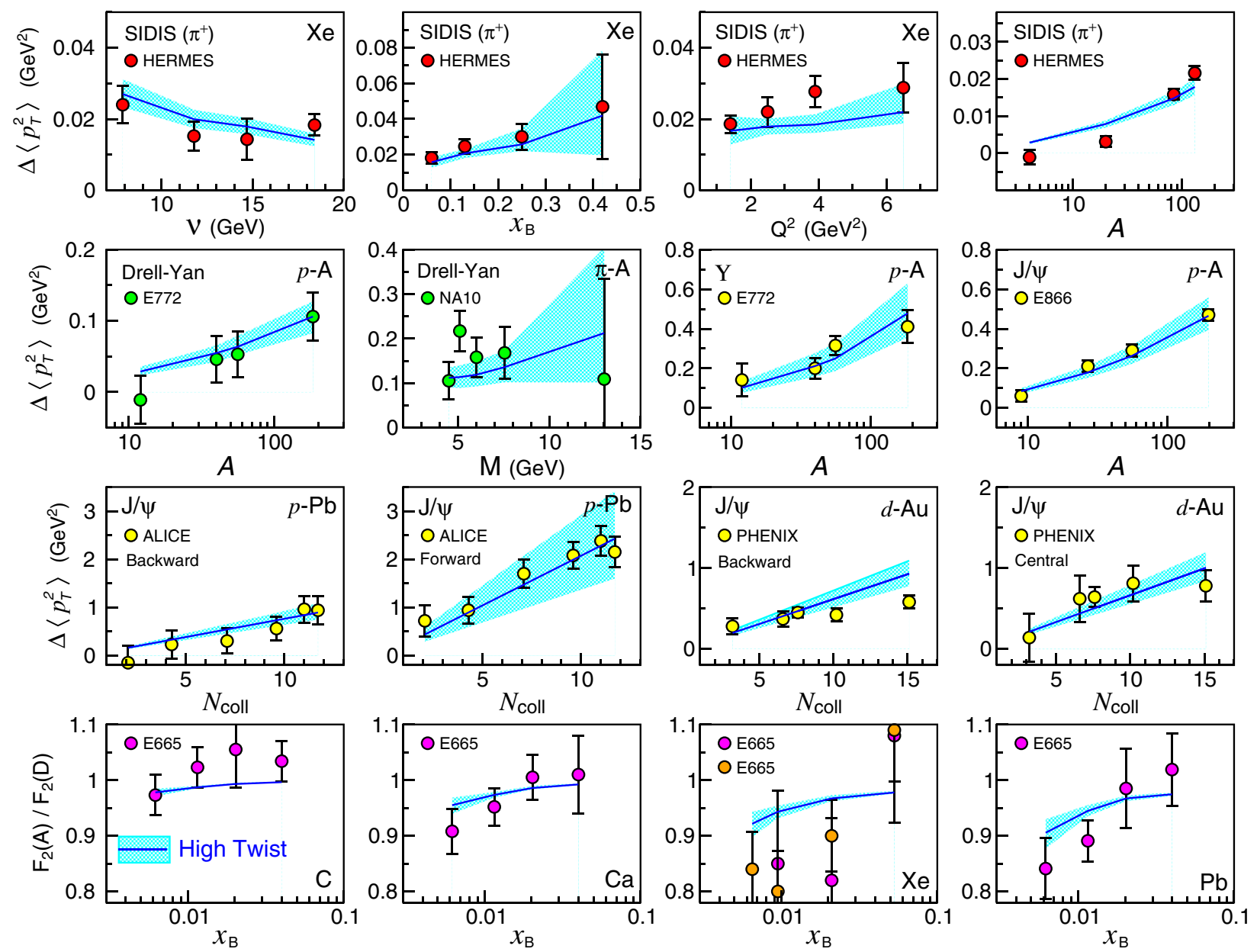

FIG. 3. The circle points correspond to the data from HERMES, E772, NA10, E772, E866, RHIC and LHC, E665. The vertical bars represent the uncertainty of experimental data (statistical and systematical uncertainties summed in quadrature). Blue curves are theoretical result from higher-twist calculation with extracted $\hat{q}$. Shaded area corresponds to theoretical uncertainty from extracted $\hat{q}$ with $90 \%$ confidence level. 


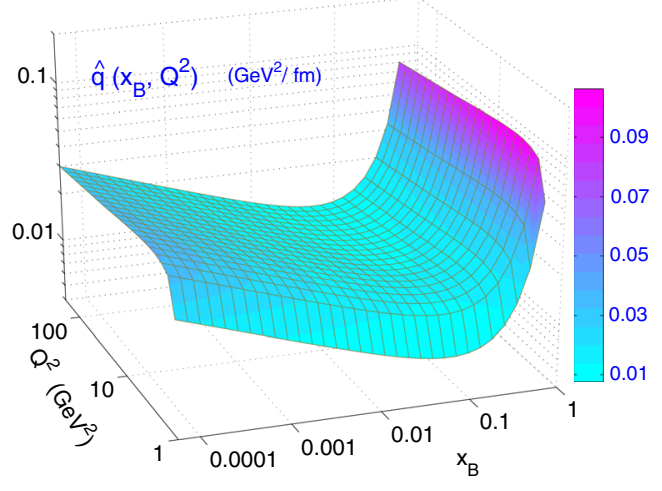

FIG. 4. The extracted $\hat{q}$ as functions of Bjorken $x_{B}$ and scale $Q^{2}$, the color bar show the $\hat{q}$ values.

observables, implying the constraining power of the different experimental measurements, in particularly with respect to $x$ and $\mu^{2}$.

We observe from Fig. 3 that the generalized factorization formalism can describe all datasets very well. This provides us with the first evidence about the universality of medium property as characterized by $\hat{q}$, and also identify quantitatively for the first time the kinematic $x$ and the probing scale $\mu^{2}$ dependence of $\hat{q}$. The $x_{B}$ and $Q^{2}$ dependences of $\hat{q}\left(x_{B}, Q^{2}\right)$ are shown in Fig. 4. The nuclear medium modification is more pronounced in small- $x$ region which is encoded in the negative power $\alpha$ in Eq. (4). This is consistent with the usual small- $x$ or gluon saturation physics, which suggests that the scattering strength or gluon density increases in the small- $x$ region [57]. On the other hand, we find that the behavior of nuclear modification in the large- $x$ region, in particularly from DIS and SIDIS data, leads to a negative power $\beta$ for $(1-x)$, and indicates an enhancement of nuclear power correction in the large- $x$ region. This seems to be consistent with the theoretical consideration as advocated in $[46,47]$. However, because of the limited data at large $x$, there is quite a large uncertainty in the extracted $\beta$ value. We hope to have more data in the future to further constrain $\hat{q}$ at larger $x$, e.g., at Jefferson Lab $12 \mathrm{GeV}$ program. Finally we find a weak scale $\mu^{2}$ dependence of $\hat{q}$. This is expected, as the logarithmic scale dependence is generally mild. As one can see from Fig. 3, the theory does give a good description of all the datasets. Nevertheless, we have to bear in mind that the fitted data has limited kinematic coverage, thus the extrapolation to small and large- $x$ regions needs to be further tested by future measurements in eA and pA collisions. The overall goodness $\chi^{2} /$ d.o.f $=1.21$ of our global fit provides informative value of $\hat{q}$ for cold nuclear matter in the intermediate $x_{B}$ and $Q^{2}$ region.

One can further infer the jet energy dependence of $\hat{q}$, which is another aspect of $\hat{q}$ under active investigation in the community. To do that, one realizes in DIS process that $x_{B}=Q^{2} / 2 m \nu$ in the target rest frame, with $\nu$ the initial jet energy. Thus the $x_{B}$ dependence of $\hat{q}$ can be extended to study the jet energy dependence of $\hat{q}$. In the intermediate $x_{B}$ and $Q^{2}$ region, the determined parametrization gives us that $\hat{q}$ increases with an increasing jet energy $\nu$. Such a behavior is consistent with the expectations of jet quenching $[5,39]$. We expect the determined parametrization form to have significant impact in precise extraction of fundamental property of QGP from jet quenching data, such as the jet energy dependence as discussed above. On the other hand, the extracted $\hat{q}$ for cold nuclear matter in this work is significantly smaller than the one extracted from the jet quenching data in relativistic heavy-ion collisions $\left(\sim 1-2 \mathrm{GeV}^{2} / \mathrm{fm}\right)[1,5-11]$, indicating the hot/dense medium created in ultrarelativistic nucleus-nucleus collisions at RHIC and the LHC has much stronger interaction strength with the hard probes than that for cold nuclear matter.

\section{SUMMARY}

We carried out the first global analysis of jet transport coefficient $\hat{q}$ for cold nuclear matter within a generalized factorization framework and a well-motivated scheme. In particular, we include the world data of transverse momentum broadening in SIDIS from HERMES, DY from SPS and FNAL, $\Upsilon$ from FNAL, and $J / \psi$ from FNAL, RHIC and LHC, comprising a total of 215 data points from 8 datasets. To check the universality of medium property, we first fit to all datasets with a constant $\hat{q}$ as commonly used in the literature. The failure of the fit in small- $x$ region strongly hints the Bjorken- $x$ and scale dependence of $\hat{q}$ and motivates us with a more advanced parametrization form. We then perform further global fit by assuming scale and $x$ dependence of $\hat{q}$ as shown in Eq. (4). The fitted parametrization form of $\hat{q}$ can describe the data very well with an overall $\chi^{2} /$ d.o.f $=1.21$. Within this scheme, our results strongly suggest the universality of medium property and provide a data driven evidence of a scale and Bjorken- $x$ dependent $\hat{q}$, which should be examined and consolidated through future theoretical and experimental studies. This parametrization form can be further extended for precise understanding of jet quenching phenomenon in relativistic heavy ion collisions.

\section{ACKNOWLEDGMENTS}

The authors thank A. Majumder and W. C. Chang for useful discussions and communications. This work is supported in part by Guangdong Major Project of Basic and Applied Basic Research No. 2020B0301030008 and by the National Natural Science Foundation of China (NSFC) under Grants No. 12022512 and No. 12035007, by Science and Technology Program of Guangzhou (No. 201905001). P. R. is supported by China Postdoctoral Science Foundation under project No. 2019M652929 and the MOE Key Laboratory of Quark and Lepton Physics (CCNU) under project No. QLPL201802.Z. K. is supported by the National Science Foundation in US under Grants No. PHY-1720486 and No. PHY-1945471. 
[1] K. M. Burke et al. (JET Collaboration), Phys. Rev. C 90, 014909 (2014).

[2] R. Baier, Y. L. Dokshitzer, A. H. Mueller, S. Peigne, and D. Schiff, Nucl. Phys. B484, 265 (1997).

[3] X.-F. Chen, T. Hirano, E. Wang, X.-N. Wang, and H. Zhang, Phys. Rev. C 84, 034902 (2011).

[4] A. Majumder and C. Shen, Phys. Rev. Lett. 109, 202301 (2012).

[5] F.-C. Zhou, G.-L. Ma, and Y.-G. Ma, Eur. Phys. J. A 56, 70 (2020).

[6] M. Xie, S.-Y. Wei, G.-Y. Qin, and H.-Z. Zhang, Eur. Phys. J. C 79, 589 (2019).

[7] G.-Y. Ma, W. Dai, B.-W. Zhang, and E.-K. Wang, Eur. Phys. J. C 79, 518 (2019).

[8] L. Chen, G.-Y. Qin, S.-Y. Wei, B.-W. Xiao, and H.-Z. Zhang, Phys. Lett. B 773, 672 (2017).

[9] C. Andres, N. Armesto, M. Luzum, C. A. Salgado, and P. Zurita, Eur. Phys. J. C 76, 475 (2016).

[10] E. Bianchi, J. Elledge, A. Kumar, A. Majumder, G. Y. Qin, and C. Shen, arXiv:1702.00481.

[11] A. Kumar, A. Majumder, and C. Shen, Phys. Rev. C 101, 034908 (2020).

[12] M. Luo, J.-w. Qiu, and G. F. Sterman, Phys. Rev. D 49, 4493 (1994).

[13] J.-w. Qiu and I. Vitev, Phys. Rev. Lett. 93, 262301 (2004).

[14] J.-w. Qiu and I. Vitev, Phys. Lett. B 632, 507 (2006).

[15] Z.-B. Kang, E. Wang, X.-N. Wang, and H. Xing, Phys. Rev. Lett. 112, 102001 (2014).

[16] Z.-B. Kang, E. Wang, X.-N. Wang, and H. Xing, Phys. Rev. D 94, 114024 (2016).

[17] Z.-B. Kang, J.-W. Qiu, X.-N. Wang, and H. Xing, Phys. Rev. D 94, 074038 (2016).

[18] J.-P. Blaizot and Y. Mehtar-Tani, Nucl. Phys. A929, 202 (2014).

[19] E. Iancu, J. High Energy Phys. 10 (2014) 095.

[20] T. Liou, A. H. Mueller, and B. Wu, Nucl. Phys. A916, 102 (2013).

[21] J.-w. Qiu and G. F. Sterman, Int. J. Mod. Phys. E 12, 149 (2003).

[22] J.-W. Qiu, Eur. Phys. J. C 43, 239 (2005).

[23] A. Airapetian et al. (HERMES Collaboration), Phys. Lett. B 684, 114 (2010).

[24] D. M. Alde et al., Phys. Rev. Lett. 66, 2285 (1991).

[25] M. J. Leitch et al., Phys. Rev. D 52, 4251 (1995).

[26] M. A. Vasilev et al. (NuSea Collaboration), Phys. Rev. Lett. 83, 2304 (1999).

[27] P. L. McGaughey, J. M. Moss, and J. C. Peng, Annu. Rev. Nucl. Part. Sci. 49, 217 (1999).

[28] J.-C. Peng, AIP Conf. Proc. 494, 503 (1999).

[29] A. Adare et al. (PHENIX Collaboration), Phys. Rev. C 87, 034904 (2013).

[30] J. Adam et al. (ALICE Collaboration), J. High Energy Phys. 11 (2015) 127.
[31] P. Bordalo et al. (NA10 Collaboration), Phys. Lett. B 193, 373 (1987).

[32] J.-w. Qiu and G. F. Sterman, Nucl. Phys. B353, 105 (1991).

[33] J.-w. Qiu and G. F. Sterman, Nucl. Phys. B353, 137 (1991).

[34] M. Luo, J.-w. Qiu, and G. F. Sterman, Phys. Rev. D 50, 1951 (1994).

[35] M. Luo, J.-w. Qiu, and G. F. Sterman, Phys. Lett. B 279, 377 (1992).

[36] M. R. Adams et al. (E665 Collaboration), Phys. Rev. Lett. 68, 3266 (1992).

[37] M. R. Adams et al. (E665 Collaboration), Z. Phys. C 67, 403 (1995).

[38] X.-f. Guo, Phys. Rev. D 58, 114033 (1998).

[39] J. Casalderrey-Solana and X.-N. Wang, Phys. Rev. C 77, 024902 (2008).

[40] R. J. Fries, Phys. Rev. D 68, 074013 (2003).

[41] Z.-B. Kang and J.-W. Qiu, Phys. Rev. D 77, 114027 (2008).

[42] Z.-B. Kang and J.-W. Qiu, Phys. Lett. B 721, 277 (2013).

[43] S. Forte, L. Garrido, J. I. Latorre, and A. Piccione, J. High Energy Phys. 05 (2002) 062.

[44] J. Collins, Foundations of Perturbative QCD (Cambridge University Press, Cambridge, England, 2011), http://www .cambridge.org/de/knowledge/isbn/item5756723.

[45] K. J. Golec-Biernat and M. Wusthoff, Phys. Rev. D 59, 014017 (1998).

[46] Y. L. Dokshitzer, G. Marchesini, and B. R. Webber, Nucl. Phys. B469, 93 (1996).

[47] S. J. Brodsky, Dynamical higher twist and high x phenomena: A window to quark quark correlations in QCD, in Workshop on Nucleon Structure in High x-Bjorken Region (HiX2000) Philadelphia, Pennsylvania (2000) [arXiv: hep-ph/0006310].

[48] F. James and M. Roos, Comput. Phys. Commun. 10, 343 (1975).

[49] X.-f. Guo and X.-N. Wang, Phys. Rev. Lett. 85, 3591 (2000).

[50] B.-W. Zhang and X.-N. Wang, Nucl. Phys. A720, 429 (2003).

[51] H. Xing, Y. Guo, E. Wang, and X.-N. Wang, Nucl. Phys. A879, 77 (2012).

[52] S. Dulat, T.-J. Hou, J. Gao, M. Guzzi, J. Huston, P. Nadolsky, J. Pumplin, C. Schmidt, D. Stump, and C.-P. Yuan, Phys. Rev. D 93, 033006 (2016).

[53] P. J. Sutton, A. D. Martin, R. G. Roberts, and W. J. Stirling, Phys. Rev. D 45, 2349 (1992).

[54] D. de Florian, R. Sassot, and M. Stratmann, Phys. Rev. D 75, 114010 (2007).

[55] D. Stump, J. Pumplin, R. Brock, D. Casey, J. Huston, J. Kalk, H. L. Lai, and W. K. Tung, Phys. Rev. D 65, 014012 (2001).

[56] J. Pumplin, D. R. Stump, and W. K. Tung, Phys. Rev. D 65, 014011 (2001).

[57] E. Iancu and R. Venugopalan, The color glass condensate and high-energy scattering in QCD, in Quark-Gluon Plasma 4, edited by R. C. Hwa and X.-N. Wang (World Scientific, Singapore, 2003), pp. 249-363. 